\title{
Effect of adjuvant chemotherapy on survival benefit in stage III colon cancer patients stratified by age: a Japanese real- world cohort study
}

\author{
Hidetaka Kawamura ${ }^{1,2}$, Toshitaka Morishima ${ }^{1 *}$ D, Akira Sato ${ }^{1}$, Michitaka Honda ${ }^{2}$ and Isao Miyashiro ${ }^{1}$
}

\begin{abstract}
Background: Adjuvant chemotherapy is relatively underused in older patients with colon cancer in Japan, and its age-specific effects on clinical outcomes remain unclear. This study aimed to assess the effect of adjuvant chemotherapy on survival benefit in stage III colon cancer patients stratified by age in a Japanese real-world setting.

Methods: In this multi-center retrospective cohort study, we analyzed patient-level information through a record linkage of population-based cancer registry data and administrative claims data. The study population comprised patients aged $\geq 18$ years who received a pathological diagnosis of stage III colon cancer and underwent curative resection between 2010 and 2014 at 36 cancer care hospitals in Osaka Prefecture, Japan. Patients were divided into two groups based on age at diagnosis ( $<75$ and $\geq 75$ years). The effect of adjuvant chemotherapy was analyzed using Cox proportional hazards regression models for all-cause mortality with inverse probability weighting of propensity scores. Adjusted hazard ratios were estimated for both age groups.
\end{abstract}

Results: A total of 783 patients were analyzed; 476 (60.8\%) were aged $<75$ years and 307 (39.2\%) were aged $\geq 75$ years. The proportion of older patients who received adjuvant chemotherapy (36.8\%) was substantially lower than that of younger patients (73.3\%). In addition, the effect of adjuvant chemotherapy was different between the age groups: the adjusted hazard ratio was 0.56 (95\% confidence interval: $0.33-0.94, P=0.027$ ) in younger patients and $1.07(0.66-1.74, P=0.78)$ in older patients.

Conclusions: The clinical effectiveness of adjuvant chemotherapy in older patients with stage III colon cancer appears limited under current utilization practices.

Keywords: Adjuvant chemotherapy, Administrative claims, Colonic neoplasms, Health services for the aged, Medical record linkage, Multicenter study, Observational study, Propensity score, Registries, Survival analysis

\section{Background}

Colon cancer is one of the most common cancers worldwide, and its incidence is increasing in the older population [1-3]. Older adults with colon cancer are generally more susceptible to issues such as impaired organ function, comorbidities, and polypharmacy $[4,5]$. These factors can contribute to a higher frequency and severity of

\footnotetext{
* Correspondence: morishima.t@oici.jp

${ }^{1}$ Cancer Control Center, Osaka International Cancer Institute, 3-1-69 Otemae, Chuo-ku, Osaka 541-8567, Japan

Full list of author information is available at the end of the article
}

adverse events in older patients undergoing medical treatment $[6,7]$.

Approximately one-fourth of incident colon cancer patients present with node-positive stage III disease [8]. Stage III colon cancer patients have a high risk of recurrence after undergoing curative resection, $[2,3]$ and several randomized controlled trials (RCTs) have compared the effectiveness of various adjuvant chemotherapy regimens for these patients [9-13]. However, clinical studies that evaluate the effects of adjuvant chemotherapy tend to have a selection bias toward younger patients. 
A pooled analysis of RCTs from the US and several European countries reported that adjuvant chemotherapy increased survival and time to tumor recurrence in older patients with stage III colon cancer [14]. Two other retrospective population-based cohort studies have also produced similar results [15, 16]. Based on this evidence, Japanese treatment guidelines currently recommend adjuvant chemotherapy for older patients with stage III colon cancer [17]. However, treatment strategies for colon cancer in Japan involve the use of complete mesocolic excision (CME) with central vascular ligation as standard procedure, which differs from the approaches used in numerous other countries [17]. Furthermore, Japan has approved the use of several types of adjuvant chemotherapy regimens for stage III colon cancer patients that are not yet approved in other countries. In addition to fluorouracil plus leucovorin (5-FU/LV), capecitabine (CAPE), 5-FU/ LV plus oxaliplatin (FOLFOX), and CAPE plus oxaliplatin (CAPEOX), adjuvant chemotherapy is also administered with uracil-tegafur plus leucovorin (UFT/LV) and tegafur/ gimeracil/oteracil/potassium (S-1). S-1 plus oxaliplatin (SOX) has also been successfully used in a clinical trial [18]. Despite the availability of these options, patients aged $\geq 75$ years with stage III colon cancer in Japan are undertreated with adjuvant chemotherapy [19]. Additionally, the effectiveness of adjuvant therapy for colon cancer in patients aged $<75$ years has been investigated in many clinical trials and the benefits of patients aged $\geq 75$ years remain unclear [11, 20, 21].

This study aimed to assess the effectiveness of postoperative adjuvant chemotherapy on survival benefit in stage III colon cancer patients stratified by age, and to characterize the types of adjuvant chemotherapy regimens used in a real-world setting in Japan.

\section{Methods}

\section{Data and cohort construction}

In this retrospective cohort study, we performed a record linkage of population-based cancer registry data and administrative data to analyze the relationship between patient mortality and clinical information that is unavailable in cancer registries. Data were obtained from a 5year period spanning January 2010 to December 2014. The study protocol was approved by the Institutional Review Board of Osaka International Cancer Institute (Approval number: 1707105108).

Cancer registry data were obtained from the Osaka Cancer Registry, a population-based registry that compiles information on cancer diagnoses and outcomes in patients residing in Osaka Prefecture (the third most populous prefecture after Tokyo and Kanagawa). Data include patient sex, age at cancer diagnosis, use or nonuse of curative resection and adjuvant chemotherapy for colon cancer, surgical approach (laparoscopic or open), vital status, and dates of death or the last follow-up to determine vital status. Tumor-specific data include primary location, degree of differentiation, date of cancer diagnosis, and Surveillance, Epidemiology, and End Results (SEER) summary stage [22].

The administrative data used in this study were produced under Japan's Diagnosis Procedure Combination (DPC) per-diem payment system, which determines insurers' reimbursements to acute care hospitals for the provision of healthcare services. These data are widely used for research in Japan [23]. DPC data comprise clinical summaries and detailed insurance claims, and include information on comorbidities, preoperative and postoperative activities of daily living (ADL), postoperative complications, and prescribed drugs. The data were obtained from 36 government-designated cancer care hospitals located throughout Osaka Prefecture.

The two data sources were linked at the patient level using each hospital's patient identification number as a linkage key [24]. This record-linked database encompassed approximately $50 \%$ of all newly diagnosed cancer cases in Osaka Prefecture during the study period.

Colon cancer cases were identified using the relevant International Classification of Diseases for Oncology, Third Edition (ICD-O3) topographical codes. Candidate subjects comprised patients aged 18 years or older who had been diagnosed with colon cancer (C18.0, C18.2-C18.8, or C19.9) at any of the 36 cancer care hospitals and had been registered in the Osaka Cancer Registry between January 1, 2010 and December 31, 2014. We focused on patients who received a diagnosis of adenocarcinoma (ICD-O3 morphological codes: 8140, 8211, 8260, 8480, 8490, or 8510) with regional lymph node metastasis according to SEER staging criteria, and had undergone curative resection for colon cancer. Regional lymph node metastasis (T1-T4a, N1-N2, M0) in the SEER staging system was ascertained in stage III (T1-T4, N1-N2, M0) patients according to the TNM classification system (version 7) of the American Joint Committee on Cancer [25]. Patients were excluded from analysis if they had missing vital status data, or had died within 90 days from the cancer diagnosis.

The exposure of interest was the use of adjuvant chemotherapy, which was defined as the administration of chemotherapy within four months after surgical resection that was included in the initial cancer treatment plan. The following seven types of adjuvant chemotherapy were identified in the DPC data for analysis: 5-FU/ LV, CAPE, UFT/LV, S-1, FOLFOX, CAPEOX, and SOX. The first three regimens are types of single-agent chemotherapy (monotherapy), and the remaining four regimens are types of double-agent chemotherapy (combined therapy). 
The endpoint was overall survival (OS), which was calculated as the number of days from the date of cancer diagnosis until the date of all-cause death, loss to followup, or alive through May 2018.

Several demographic and clinical variables were obtained for analysis. These included sex, age at diagnosis $(<75$ and $\geq$ 75 years), and degree of tumor differentiation (well-, moderately, and poorly differentiated). In addition, tumor localization was divided into two anatomical subsites: right colon (ICD-O3 topographical codes: C18.0 and C18.2C18.5) and left colon (C18.6-C18.8 and C19.9). Body mass index was classified into four categories $(<18.5$, 18.5-24.9, $25.0-29.9$, and $\geq 30 \mathrm{~kg} / \mathrm{m}^{2}$ ). The Charlson comorbidity index (CCI) was used to measure patients' comorbidities on admission for radical surgery for colon cancer, $[26,27]$ and was classified into four categories based on CCI scores $(0,1,2$, or $\geq 3$ ). The Barthel index was used to measure patients' ADL on admission and discharge for radical surgery for colon cancer; this index uses a scale from 0 to 100 , and was classified into five categories $(0-20,21-60,61-90,90-99$, 100) for analysis [28]. The following postoperative complications were identified from the relevant data fields in the DPC file using International Classification of Diseases, Tenth Revision codes: surgical site infection (T79.3 or T81.4), peritonitis or peritoneal abscess (K65.x), pancreatic injury (K91.8), ileus (K56.x or K91.3), anastomotic stenosis (T81.8), sepsis (A40.x or A41.x), respiratory complication (pneumonia [J12.xJ18.x], postprocedural respiratory disorder [J95.x], or respiratory failure [J96.x]), pulmonary embolism (I26.x), cardiac event (acute coronary event [I21.x-I24.x] or heart failure [I50.x]), cerebral infarction or hemorrhage (I60.x-I64.x), acute renal failure $(\mathrm{N} 17 . \mathrm{x})$, and urinary tract infection (N10.x, N30.x, or N39.0) [29].

\section{Statistical analyses}

The clinical factors and adjuvant chemotherapy regimens for each of the patient age groups $(<75$ and $\geq 75$ years) were analyzed using descriptive statistics. We examined frequencies for categorical variables and medians with interquartile range (IQR) for continuous variables. Univariate analyses were employed to compare the variables between the two patient age groups, in which categorical variables were compared with Fisher's exact test and continuous variables were compared with the Mann-Whitney $U$ test.

Survival analysis was performed using the Kaplan-Meier method, and survival estimates were compared using the log-rank test. The effect of adjuvant chemotherapy on OS was analyzed using Cox proportional hazards regression models for all-cause mortality with inverse probability weighting based on propensity scores to adjust for differences between the postoperative treatment groups (surgery with adjuvant chemotherapy and surgery alone) [30]. In order to determine the inverse probability of treatment weights, each patient's propensity score was calculated using logistic regression to estimate the probability of receiving adjuvant chemotherapy conditional to his/her characteristics: sex, age at diagnosis, degree of tumor differentiation, radical surgery procedure, tumor location, body mass index, preoperative and postoperative Barthel index, CCI, and postoperative complications. We then assigned patients who underwent postoperative adjuvant chemotherapy a weight of $1 \div$ (propensity score) and those who underwent surgery alone a weight of $1 \div(1$ - propensity score). We assessed covariate balance using absolute standardized differences; a difference of $10 \%$ or less was considered to indicate a well-balanced result [31]. Adjusted hazard ratios (HRs) and 95\% confidence intervals (CIs) were estimated for each patient age group. Missing data were imputed using the multiple imputation method [32]. We also estimated adjusted HRs using complete data (without missing data imputation) as a sensitivity analysis.

All significance tests were 2 -sided and $P$ values $<0.05$ were considered to be statistically significant. Statistical analyses were performed using STATA version 15.1 software (STATA Corporation, College Station, TX, USA).

The datasets used and/or analyzed during the current study are available from the corresponding author on reasonable request.

\section{Results}

We identified 783 eligible patients aged 18 years or older who underwent curative resection and were diagnosed with stage III colon cancer between 2010 and 2014 (Fig. 1). Of these patients, $476(60.8 \%)$ were aged $<75$ years (younger patients), and 307 patients (39.2\%) were aged $\geq 75$ years (older patients). A total of 349 younger patients $(73.3 \%)$ and 113 older patients (36.8\%) received adjuvant chemotherapy after surgery. The median follow-up time was 1495 days (IQR: 1315-1979 days), and 192 patients $(24.5 \%)$ died during follow-up.

The patient characteristics are summarized in Table 1. The median age was 66 years (IQR: $60-71$ years) in the younger patients and 80 years (IQR: $76-83$ years) in the older patients. When compared with older patients, a higher percentage of younger patients had tumors in the left colon $(64.1 \%$ vs $48.0 \% ; P=0.001)$, no comorbidities $(\mathrm{CCI}=0) \quad(79.0 \%$ vs $57.9 \% ; P<0.001)$, and good preoperative (93.3\% vs $77.9 \% ; P<0.001)$ and postoperative (95.0\% vs 79.1\%; $P<0.001)$ ADL (Barthel index $=100$ ). In addition, a lower percentage of younger patients had postoperative complications $(8.4 \%$ vs $12.8 \% ; P=0.022)$.

Table 2 shows the distribution of adjuvant chemotherapy regimens in all patients and the two age groups. Among the 462 patients who received adjuvant chemotherapy, 298 patients $(64.5 \%)$ received monotherapy and 164 patients $(35.5 \%)$ received combined therapy. UFT/ $\mathrm{LV}$ was the most commonly used regimen $(n=139$ 


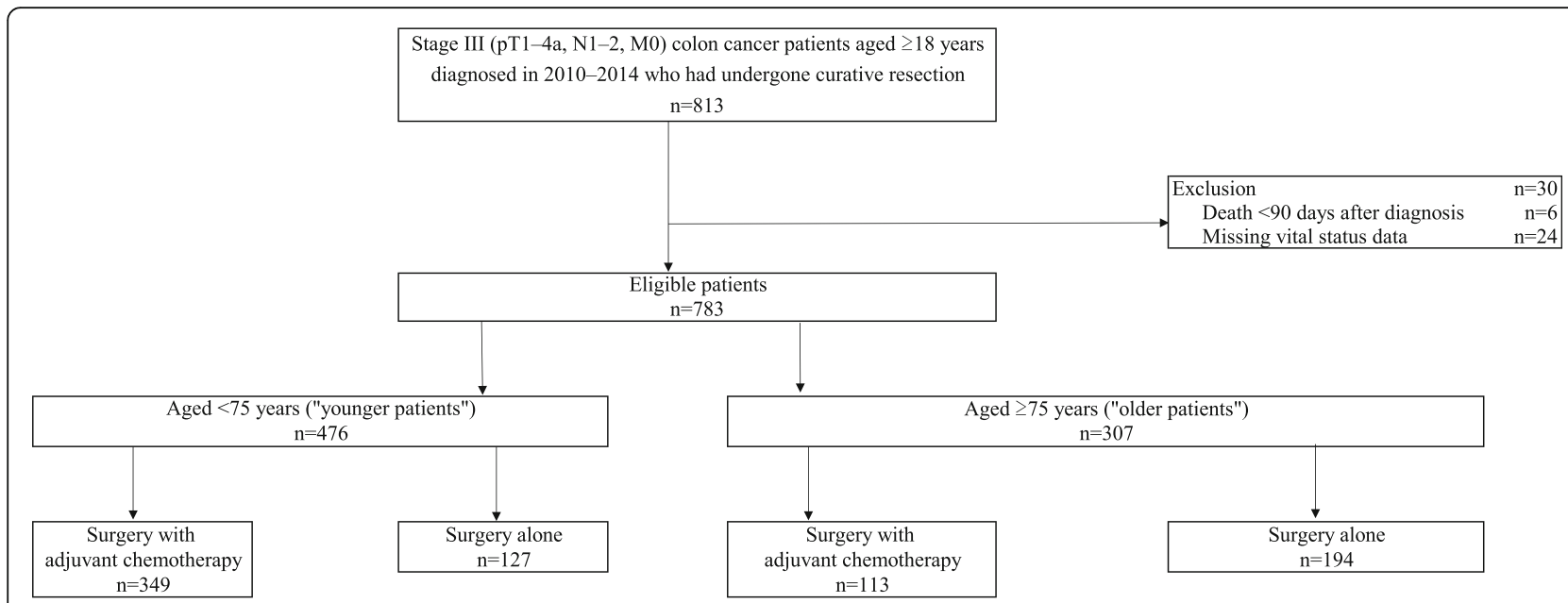

Fig. 1 Overview of patient selection and use of adjuvant chemotherapy stratified by age

[30.1\%]), especially in older patients $(n=47$ [41.6\%]). The percentage of younger patients who received combined therapy was approximately twice that of older patients $(40.7 \%$ vs $19.5 \%)$.

OS analysis was performed using the Kaplan-Meier method (Fig. 2). For younger patients (Fig. 2a), the 3year estimated OS for surgery with adjuvant chemotherapy and surgery alone was $94.2 \%$ (95\% CI: 91.1-96.2\%) and $87.1 \%$ (95\% CI: 79.8-91.9\%), respectively $(P=0.002)$. Among these patients, the 5-year estimated OS for surgery with adjuvant chemotherapy and surgery alone was 84.6\% (95\% CI: 79.1-88.7\%) and 77.1\% (95\% CI: 67.9$84.0 \%)$, respectively $(P=0.002)$. For older patients (Fig. $2 \mathrm{~b})$, the 3-year estimated OS for surgery with adjuvant chemotherapy and surgery alone was $82.9 \%$ (95\% CI: 74.5-88.7\%) and 78.0\% (95\% CI: 71.5-83.3\%), respectively $(P=0.002)$. Among these patients, the 5 -year estimated OS for surgery with adjuvant chemotherapy and surgery alone was $74.2 \%$ (95\% CI: $64.0-81.9 \%$ ) and 53.1\% (95\% CI: $44.1-61.3 \%)$, respectively $(P=0.002)$.

The effect of adjuvant chemotherapy on survival benefit was estimated using Cox proportional hazards regression models for all-cause mortality with inverse probability weighting of propensity scores (Table 3). The covariates were well-balanced in the propensity-weighted cohort, with all standardized differences below 10\% (data not shown). For younger patients, the adjusted HR of surgery with adjuvant chemotherapy compared with surgery alone was 0.56 (95\% CI: $0.33-0.94, P=0.027$ ). For older patients, the adjusted HR of surgery with adjuvant chemotherapy compared with surgery alone was 1.07 (95\% CI: $0.66-1.74, P=0.78$ ). The sensitivity analysis with complete data similarly showed different effects of adjuvant chemotherapy between these groups: the adjusted HR of surgery with adjuvant chemotherapy was 0.55 (95\% CI: $0.33-0.94, P=0.028$ ) in younger patients and 1.13 (95\% CI: $0.71-1.81, P=0.60$ ) in older patients.

\section{Discussion}

To our knowledge, this represents one of the largest Japanese real-world studies to date that evaluates the effectiveness of adjuvant chemotherapy after surgical resection in older patients with stage III colon cancer that accounts for potential confounding factors [19]. The main study findings are as follows: although adjuvant chemotherapy demonstrated a significant association with improved survival among younger patients aged < 75 years, no such association was observed among older patients aged $\geq 75$ years. Next, the utilization rate of adjuvant chemotherapy was $59 \%$ among all patients; of these, $64.5 \%$ received monotherapy and $35.5 \%$ received combined therapy. UFT/LV was the most commonly used regimen. In addition, the 5-year OS for older patients with stage III colon cancer for surgery with adjuvant chemotherapy and surgery alone was 74.2 and $53.1 \%$, respectively.

The lack of a significant association between adjuvant chemotherapy and longer survival in older patients could be due to a variety of factors, such as patient selection, the relatively small sample size, the types of adjuvant chemotherapy used, and unmeasured confounding. While younger patients who received adjuvant chemotherapy had longer survival times than those who underwent surgery alone, the benefits of adjuvant chemotherapy appeared to be smaller in older patients in a real-world setting. Additionally, the log-rank test indicated that adjuvant chemotherapy was associated with longer survival in older patients, but the Cox proportional hazard model showed no statistical significance and an HR $>1$ for mortality. We posit that this small increase in HR may have been influenced by the presence of unmeasured confounding factors, such as lymph node metastasis (an important prognostic factor for stage III colon cancer), as this information was not included in the study data. Furthermore, 
Table 1 Patient characteristics

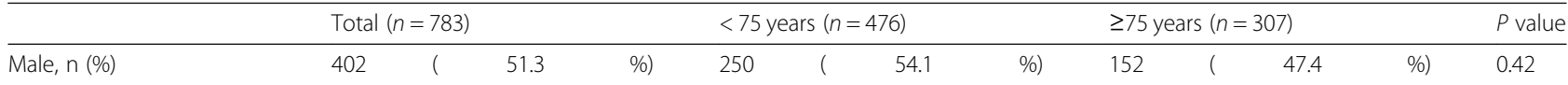

Age in years, median (IQR) 72

Body mass index in $\mathrm{kg} / \mathrm{m}^{2}, \mathrm{n}(\%)$

\begin{tabular}{|c|c|c|c|c|c|c|c|c|c|c|c|c|}
\hline$<18.5$ & 88 & ( & 11.2 & $\%)$ & 50 & ( & 10.8 & \%) & 38 & ( & 11.8 & $\%$ \\
\hline $18.5-24.9$ & 523 & ( & 66.8 & $\%)$ & 321 & ( & 69.5 & \%) & 202 & ( & 62.9 & $\%$ \\
\hline $25.0-29.9$ & 137 & ( & 17.5 & $\%)$ & 85 & ( & 18.4 & \%) & 52 & ( & 16.2 & $\%$ \\
\hline$\geq 30$ & 25 & ( & 3.2 & $\%)$ & 17 & ( & 3.7 & $\%)$ & 8 & ( & 2.5 & $\%$ \\
\hline Unknown & 10 & ( & 1.3 & $\%)$ & 3 & ( & 0.6 & $\%)$ & 7 & ( & 2.2 & $\%$ \\
\hline
\end{tabular}

Tumor differentiation, n (\%)

\begin{tabular}{|c|c|c|c|c|c|c|c|c|c|c|c|c|}
\hline Well & 188 & ( & 24.0 & $\%)$ & 110 & ( & 23.8 & \%) & 78 & ( & 24.3 & \%) \\
\hline Moderate & 499 & ( & 63.7 & $\%)$ & 309 & ( & 66.9 & $\%)$ & 190 & ( & 59.2 & $\%)$ \\
\hline Poor & 29 & ( & 3.7 & $\%)$ & 14 & ( & 3.0 & \%) & 15 & ( & 4.7 & $\%)$ \\
\hline Unknown & 67 & ( & 8.6 & $\%)$ & 43 & ( & 9.3 & $\%)$ & 24 & ( & 7.5 & $\%)$ \\
\hline
\end{tabular}

Tumor location, n (\%)

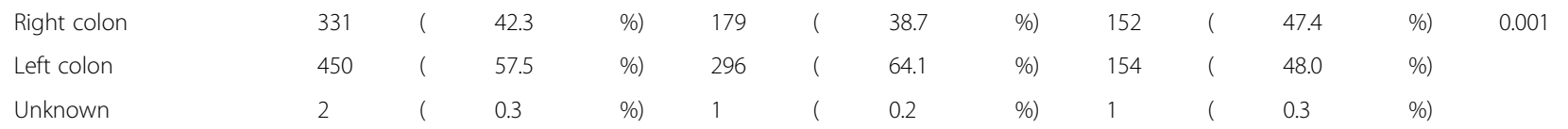

Charlson comorbidity index, $\mathrm{n}(\%)$

\begin{tabular}{|c|c|c|c|c|c|c|c|c|c|c|c|c|c|}
\hline 0 & 551 & ( & 70.4 & $\%)$ & 365 & ( & 79.0 & \%) & 186 & ( & 57.9 & $\%)$ & $<0.001$ \\
\hline 1 & 40 & ( & 5.1 & $\%)$ & 20 & ( & 4.3 & \%) & 20 & t & 6.2 & $\%)$ & \\
\hline 2 & 111 & ( & 14.2 & $\%)$ & 48 & 1 & 10.4 & \%) & 63 & ( & 19.6 & $\%)$ & \\
\hline$\geq 3$ & 81 & ( & 10.3 & \%) & 43 & ( & 9.3 & $\%)$ & 38 & ( & 11.8 & $\%)$ & \\
\hline
\end{tabular}

Preoperative Barthel index, $\mathrm{n}(\%)$

\begin{tabular}{|c|c|c|c|c|c|c|c|c|c|c|c|c|c|}
\hline $0-20$ & 19 & ( & 2.4 & $\%)$ & 6 & ( & 1.3 & $\%)$ & 13 & ( & 4.0 & $\%)$ & $<0.001$ \\
\hline $21-60$ & 30 & ( & 3.8 & $\%)$ & 9 & ( & 1.9 & \%) & 21 & ( & 6.5 & \%) & \\
\hline $61-90$ & 40 & ( & 5.1 & $\%)$ & 24 & ( & 5.2 & $\%)$ & 16 & ( & 5.0 & $\%)$ & \\
\hline $91-99$ & 12 & ( & 1.5 & $\%)$ & 6 & ( & 1.3 & \%) & 6 & ( & 1.9 & \%) & \\
\hline 100 & 681 & ( & 87.0 & $\%)$ & 431 & ( & 93.3 & \%) & 250 & ( & 77.9 & \%) & \\
\hline Unknown & 1 & ( & 0.1 & $\%)$ & 0 & ( & 0.0 & $\%)$ & 1 & ( & 0.3 & $\%)$ & \\
\hline
\end{tabular}

Surgical approach, n (\%)

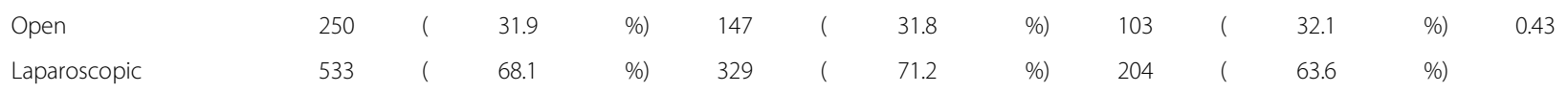

Postoperative complications, n (\%)

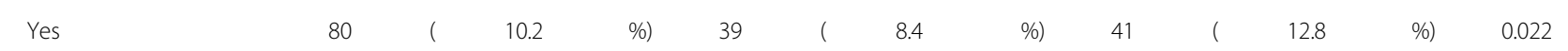

Postoperative Barthel index, $\mathrm{n}(\%)$

\begin{tabular}{|c|c|c|c|c|c|c|c|c|c|c|c|c|c|}
\hline $0-20$ & 9 & ( & 1.1 & $\%)$ & 2 & ( & 0.4 & $\%)$ & 7 & ( & 2.2 & $\%)$ & $<0.001$ \\
\hline $21-60$ & 28 & ( & 3.6 & $\%)$ & 7 & ( & 1.5 & $\%)$ & 21 & ( & 6.5 & $\%)$ & \\
\hline $61-90$ & 39 & ( & 5.0 & $\%)$ & 21 & ( & 4.5 & $\%)$ & 18 & ( & 5.6 & $\%)$ & \\
\hline $91-99$ & 13 & ( & 1.7 & $\%)$ & 7 & ( & 1.5 & $\%)$ & 6 & ( & 1.9 & $\%)$ & \\
\hline 100 & 693 & ( & 88.5 & $\%)$ & 439 & ( & 95.0 & $\%)$ & 254 & ( & 79.1 & $\%)$ & \\
\hline Unknown & 1 & ( & 0.1 & $\%)$ & 0 & ( & 0.0 & $\%)$ & 1 & ( & 0.3 & $\%)$ & \\
\hline \multicolumn{14}{|c|}{ Adjuvant chemotherapy } \\
\hline Yes & 462 & ( & 59.0 & $\%)$ & 349 & ( & 73.3 & $\%)$ & 113 & ( & 36.8 & $\%)$ & $<0.001$ \\
\hline
\end{tabular}


Table 2 Distribution of adjuvant chemotherapy regimens among patients

\begin{tabular}{|c|c|c|c|c|c|c|c|c|c|c|c|c|c|}
\hline \multirow[b]{3}{*}{ Single-agent chemotherapy } & \multicolumn{4}{|c|}{ Total $(n=462)$} & \multicolumn{4}{|c|}{$<75$ years $(n=349)$} & \multicolumn{4}{|c|}{$\geq 75$ years $(n=113)$} & \multirow{2}{*}{$\begin{array}{l}P \\
\text { value }\end{array}$} \\
\hline & \multicolumn{2}{|l|}{$n$} & \multicolumn{2}{|l|}{$\%$} & \multicolumn{2}{|l|}{$n$} & \multicolumn{2}{|l|}{$\%$} & \multicolumn{2}{|l|}{$\mathrm{n}$} & \multicolumn{2}{|l|}{$\%$} & \\
\hline & 298 & ( & 64.5 & $\%)$ & 207 & ( & 59.3 & $\%)$ & 91 & ( & 80.5 & $\%)$ & $<0.001$ \\
\hline 5-FU/LV & 13 & ( & 2.8 & $\%)$ & 11 & ( & 3.2 & $\%)$ & 2 & ( & 1.8 & $\%)$ & \\
\hline CAPE & 100 & ( & 21.6 & $\%)$ & 75 & ( & 21.5 & $\%)$ & 25 & ( & 22.1 & $\%)$ & \\
\hline UFT/LV & 139 & ( & 30.1 & $\%)$ & 92 & ( & 26.4 & $\%)$ & 47 & ( & 41.6 & $\%)$ & \\
\hline S-1 & 46 & ( & 10.0 & $\%)$ & 29 & ( & 8.3 & $\%)$ & 17 & ( & 15.0 & $\%)$ & \\
\hline Double-agent chemotherapy & 164 & ( & 35.5 & $\%)$ & 142 & ( & 40.7 & $\%)$ & 22 & ( & 19.5 & $\%)$ & \\
\hline FOLFOX & 35 & ( & 7.6 & $\%)$ & 26 & ( & 7.4 & $\%)$ & 9 & ( & 8.0 & $\%)$ & \\
\hline CAPEOX & 126 & ( & 27.3 & $\%)$ & 114 & ( & 32.7 & $\%)$ & 12 & ( & 10.6 & $\%)$ & \\
\hline sox & 3 & ( & 0.6 & $\%)$ & 2 & ( & 0.6 & $\%)$ & 1 & ( & 0.9 & $\%)$ & \\
\hline
\end{tabular}

5-FU/LV, fluorouracil plus leucovorin; CAPE, capecitabine; UFT/LV, uracil-tegafur plus leucovorin; S-1, tegafur/gimeracil/oteracil/potassium; FOLFOX, 5-FU/LV plus oxaliplatin; CAPEOX, CAPE plus oxaliplatin; SOX, S-1 plus oxaliplatin

other unmeasured confounders such as socioeconomic status (which is a risk factor for poor survival among cancer patients and is associated with a lower frequency of adjuvant chemotherapy use) are likely to create a bias in favor of adjuvant chemotherapy. In this way, the observed effect of adjuvant chemotherapy for older patients with stage III colon cancer may be smaller than expected. Because this study showed no significant difference in the effect of adjuvant chemotherapy in older patients, further analyses are needed to verify these findings and to explore the possible underlying causes.

The standard surgical treatment of colon cancer in Japan involves CME with central vascular ligation, which differs from that of many European countries and the US. CME is based on the principles of total mesorectal excision, where the entire embryological mesocolon (from the viscera to the parietal planes) is excised horizontally. This procedure has been reported to improve long-term outcomes in colon cancer without major surgical risks $[33,34]$. On the other hand, the effects of central vascular ligation are more controversial [35]. In Japan, this technique has been employed as a standard surgical procedure for colon cancer for the several decades. In contrast, countries in Europe and the US proposed CME as a standardized surgical technique in 2009, but central vascular ligation remains a nonstandardized procedure. These differences in surgical procedures should be kept in mind when interpreting and comparing the findings of studies on the effects of adjuvant chemotherapy.

Previous studies have reported similar effects of adjuvant chemotherapy for both younger and older patients [14-16]. However, these studies have included few, if any, Asian patients. Other articles have reported that the prognosis of Asian people with colon cancer is better than non-Asians [36, 37]. This racial difference may affect the age-dependent benefits of adjuvant chemotherapy. A Japanese study previously examined the effectiveness of adjuvant chemotherapy in older patients using data from the Japanese Study Group for Postoperative Follow-up of Colorectal Cancer, which comprises colorectal cancer treatment experts in Japan; that study concluded that adjuvant chemotherapy is effective in improving OS in older patients with stage III colon cancer [19]. However, their study was conducted using data from 2004 to 2006, and only accounted for a few confounding factors. Furthermore, the popularization of laparoscopic surgery during that period may have helped to reduce postoperative complications. These factors may have contributed to that study's conclusion that adjuvant chemotherapy is effective for older patients with stage III colon cancer.

The utilization rate of adjuvant chemotherapy was 59\% among all patients (monotherapy: 64.5\%, combined therapy: $35.5 \%$ ). A previous study reported that $44.8 \%$ of cancer patients in Japan received adjuvant chemotherapy [38]. In this way, the proportion of Japanese patients who receive adjuvant chemotherapy (especially combined therapy) is generally lower than that reported in other countries $[39,40]$. This relatively infrequent use of adjuvant chemotherapy may affect its perceived benefits in older Japanese patients with stage III colon cancer. In addition, the lack of adjuvant chemotherapy (especially combined therapy) may contribute to poorer prognoses in these patients.

UFT/LV was the most common type of adjuvant chemotherapy administered in our subjects, particularly in the older patients. In addition, $15 \%$ of older patients received S-1. In contrast, other countries have reported CAPE to be the most popular oral 5 -FU prodrug [41, 42]. In Japan, however, oral 5-FU derivatives are preferred because of their convenience, leading to the development of several oral 5-FU derivatives with different properties, such as UFT/LV and S-1. UFT/LV is commonly used for stage III colon cancer patients in Japan because its non-inferiority to intravenous $5-\mathrm{FU} / \mathrm{LV}$ has 


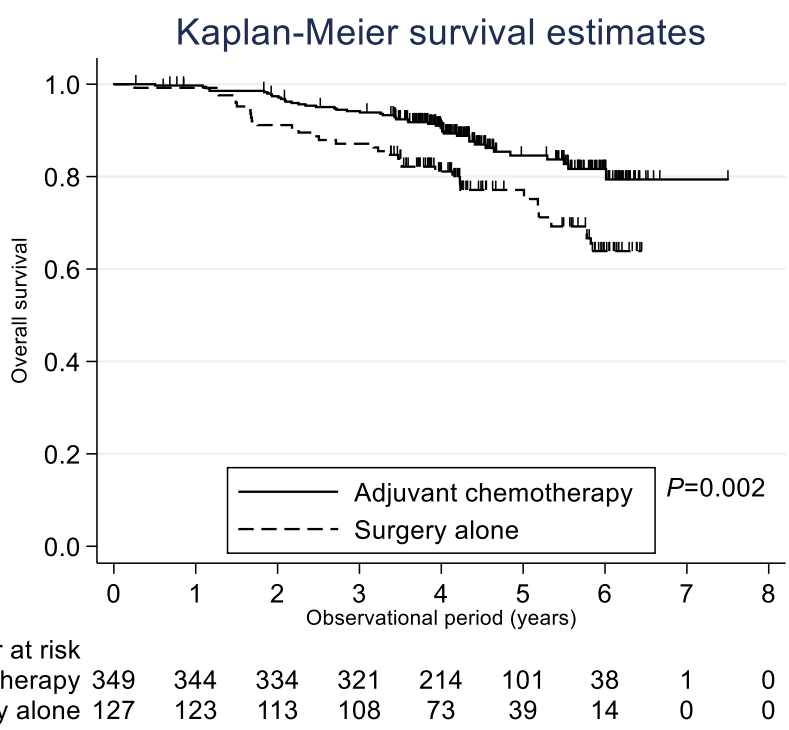

(a)

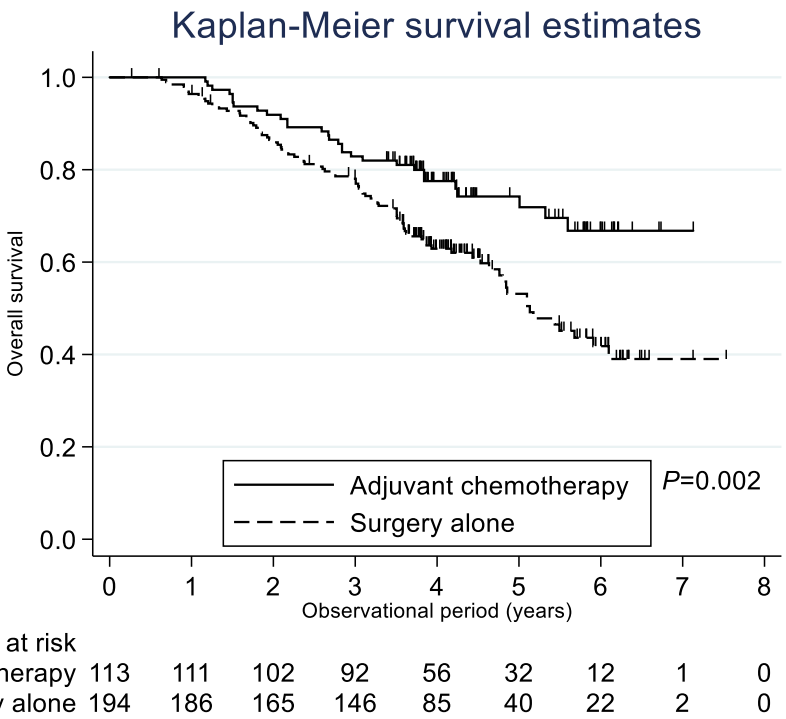

(b)

Fig. 2 Overall survival according to the use or non-use of adjuvant chemotherapy in (a) patients aged $<75$ and (b) patients aged $\geq 75$ years

been confirmed by RCTs $[11,43]$. Another trial determined that S-1 for stage III colon cancer is non-inferior to UFT/LV [12]. However, it remains unclear as to which oral 5 -FU is the most effective, especially in older patients [13].

These findings should be interpreted with consideration to several limitations. Firstly, we could not investigate the severity of lymph node metastasis-which is an important prognostic factor for stage III colon cancer-as this information was not included in the study data. Secondly, this study did not include any patients with stage T4b cancer (involving the direct invasion of adjacent organs), which is also an important negative prognostic factor. The lack of such patients could result in selection bias. Thirdly, we could not investigate the cause of death, presence or absence of recurrence, adverse events of adjuvant chemotherapy, or the quality of surgery due to data limitations. Furthermore, the data lacked detailed clinical information (such as lymph node metastasis or the reasons for each patient's treatment plan), which is an intrinsic limitation of using cancer registry data. As a result, there may still be unmeasured confounding factors in the analysis. Next, the sample size of this study may still be relatively small despite being conducted in a real-world setting in one of 
Table 3 Adjusted hazard ratios of all-cause mortality using Cox proportional hazards regression models with inverse probability weighting of the propensity score

\begin{tabular}{|c|c|c|c|c|c|c|}
\hline & \multicolumn{3}{|c|}{ Multiple imputation of missing data } & \multicolumn{3}{|c|}{ Complete data analysis } \\
\hline & Adjusted HR & $95 \% \mathrm{Cl}$ & $P$ value & Adjusted HR & $95 \% \mathrm{Cl}$ & $P$ value \\
\hline \multicolumn{7}{|l|}{$<75$ years } \\
\hline Surgery alone & (reference) & & & (reference) & & \\
\hline Surgery with adjuvant chemotherapy & 0.56 & $(0.33-0.94)$ & 0.027 & 0.55 & $(0.33-0.94)$ & 0.028 \\
\hline \multicolumn{7}{|l|}{$\geq 75$ years } \\
\hline Surgery alone & (reference) & & & (reference) & & \\
\hline Surgery with adjuvant chemotherapy & 1.07 & $(0.66-1.74)$ & 0.78 & 1.13 & $(0.71-1.81)$ & 0.60 \\
\hline
\end{tabular}

Adjusted for sex, age at diagnosis, degree of tumor differentiation, radical surgery procedure, tumor location, body mass index, preoperative and postoperative Barthel index, comorbidities, and postoperative complications

$\mathrm{Cl}$, confidence interval; $\mathrm{HR}$, hazard ratio

Japan's largest prefectures. This may also contribute to selection bias. The inclusion of other types of registry data may enable further investigations into these limitations. Finally, we were unable to examine if the effects of adjuvant chemotherapy were attributable to single-agent chemotherapy or double-agent chemotherapy because the study's primary objective was to determine whether adjuvant chemotherapy was associated with improved survival irrespective of regimen type. Further studies are needed to identify the optimal regimen for stage III colon cancer patients in the real-world setting.

This study is, to the best of our knowledge, the first Japanese real-world cohort study that uses a combination of cancer registry data and administrative data to examine the survival benefit of adjuvant chemotherapy in stage III colon cancer patients. In clinical practice, clinicians often struggle with treatment strategies for subpopulations that are generally not included in RCTs, such as older patients. The real-world setting of this study is useful for the resolution of this issue. Additionally, the combination of two different datasets helped to overcome the limitations of each data source. Because the Japanese treatment strategies for stage III colon cancer patients have demonstrated effectiveness in several RCTs, [11-13, 18, 43] our findings are potentially generalizable to hospitals that employ these strategies.

\section{Conclusions}

Adjuvant chemotherapy did not demonstrate any significant effects on survival benefit in older patients with stage III colon cancer under current utilization practices. These real-world findings may have applications in informing the treatment strategies of older patients, who are frequently not included in the population of RCTs.

\section{Abbreviations}

5-FU/LV: Fluorouracil plus leucovorin; ADL: Activities of daily living; CAPE: Capecitabine; CAPEOX: CAPE plus oxaliplatin; CCl: Charlson comorbidity index; Cl: Confidence interval; CME: Complete mesocolic excision; DPC: Diagnosis Procedure Combination; FOLFOX: 5-FU/LV plus oxaliplatin; HR: Hazard ratio; ICD-O3: International Classification of Diseases for Oncology, Third Edition; IQR: Interquartile range; OS: Overall survival; RCT: Randomized controlled trial; S-1: Oral fluoropyrimidine; SEER: Surveillance, Epidemiology, and End Results; SOX: S-1 plus oxaliplatin; UFT/LV: Uracil-tegafur plus leucovorin

\section{Acknowledgements}

We would like to express our gratitude to the 36 cancer care hospitals in Osaka Prefecture for providing the DPC data and helping with the record linkage, and to Dr. Takahiro Higashi (National Cancer Center, Japan) for his technical assistance and advice on the record linkage process.

\section{Authors' contributions}

HK analyzed and interpreted the data and was a major contributor in writing the manuscript. TM and AS obtained and interpreted the data. MH interpreted the data. IM obtained the data. All authors read and approved the final manuscript.

\section{Funding}

This work was supported by a research grant from the Health Care Science Institute (Grant Number 28-9) and a Grant-in-Aid for Young Scientists from the Japan Society for the Promotion of Science (JSPS KAKENHI Grant Number JP16K19291). The funders had no role in the study design; the collection, analysis and interpretation of data; the writing of the report; or the decision to submit the article for publication.

\section{Availability of data and materials}

The datasets used and/or analyzed during the current study are available from the corresponding author on reasonable request.

\section{Ethics approval and consent to participate}

The study protocol was approved by the Institutional Review Board of Osaka International Cancer Institute (Approval number: 1707105108). This board waived the need for informed consent in accordance with the Japanese government's Ethical Guidelines for Medical and Health Research Involving Human Subjects, which allow for the opt-out approach for the secondary use of existing data.

\section{Consent for publication}

Not applicable.

\section{Competing interests}

The authors declare that they have no competing interests.

\section{Author details}

${ }^{1}$ Cancer Control Center, Osaka International Cancer Institute, 3-1-69 Otemae, Chuo-ku, Osaka 541-8567, Japan. ${ }^{2}$ Department of Minimally Invasive Surgical and Medical Oncology, Fukushima Medical University, Fukushima, Japan. 


\section{Received: 18 August 2019 Accepted: 30 December 2019} Published online: 06 January 2020

\section{References}

1. Ferlay J, Soerjomataram I, Dikshit R, Eser S, Mathers C, Rebelo M, et al. Cancer incidence and mortality worldwide: sources, methods and major patterns in GLOBOCAN 2012. Int J Cancer. 2015;136:E359-86.

2. Miller KD, Siegel RL, Lin CC, Mariotto AB, Kramer JL, Rowland JH, et al. Cancer treatment and survivorship statistics, 2016. CA Cancer J Clin. 2016;66:271-89.

3. Nitsche U, Spath C, Muller TC, Maak M, Janssen KP, Wilhelm D, et al. Colorectal cancer surgery remains effective with rising patient age. Int J Color Dis. 2014;29:971-9.

4. Millan M, Merino S, Caro A, Feliu F, Escuder J, Francesch T. Treatment of colorectal cancer in the elderly. World J Gastrointest Oncol. 2015;7:204-20.

5. Yancik R, Wesley MN, Ries LA, Havlik RJ, Long S, Edwards BK, et al. Comorbidity and age as predictors of risk for early mortality of male and female colon carcinoma patients: a population-based study. Cancer. 1998; 82:2123-34

6. Studenski S, Perera S, Patel K, Rosano C, Faulkner K, Inzitari M, et al. Gait speed and survival in older adults. JAMA. 2011;305:50-8.

7. Charlson ME, Pompei P, Ales KL, MacKenzie CR. A new method of classifying prognostic comorbidity in longitudinal studies: development and validation. J Chronic Dis. 1987;40:373-83.

8. Hori M, Matsuda T, Shibata A, Katanoda K, Sobue T, Nishimoto H. Cancer incidence and incidence rates in Japan in 2009: a study of 32 populationbased cancer registries for the monitoring of Cancer incidence in Japan (MCIJ) project. Jpn J Clin Oncol. 2015;45:884-91.

9. Andre T, Boni C, Mounedji-Boudiaf L, Navarro M, Tabernero J, Hickish T, et al. Oxaliplatin, fluorouracil, and leucovorin as adjuvant treatment for colon cancer. N Engl J Med. 2004;350:2343-51.

10. Haller DG, Tabernero J, Maroun J, de Braud F, Price T, Van Cutsem E, et al. Capecitabine plus oxaliplatin compared with fluorouracil and folinic acid as adjuvant therapy for stage III colon cancer. J Clin Oncol. 2011;29:1465-71.

11. Shimada Y, Hamaguchi T, Mizusawa J, Saito N, Kanemitsu Y, Takiguchi N, et al. Randomised phase III trial of adjuvant chemotherapy with oral uracil and tegafur plus leucovorin versus intravenous fluorouracil and levofolinate in patients with stage III colorectal cancer who have undergone Japanese D2/D3 lymph node dissection: final results of JCOG0205. Eur J Cancer. 2014; 50:2231-40.

12. Yoshida M, Ishiguro M, Ikejiri K, Mochizuki I, Nakamoto $Y$, Kinugasa $Y$, et al. S-1 as adjuvant chemotherapy for stage III colon cancer: a randomized phase III study (ACTS-CC trial). Ann Oncol. 2014;25:1743-9.

13. Hamaguchi T, Shimada Y, Mizusawa J, Kinugasa Y, Kanemitsu Y, Ohue M, et al. Capecitabine versus S-1 as adjuvant chemotherapy for patients with stage III colorectal cancer (JCOG0910): an open-label, non-inferiority, randomised, phase 3, multicentre trial. Lancet Gastroenterol Hepatol. 2018;3:47-56.

14. Sargent DJ, Goldberg RM, Jacobson SD, Macdonald JS, Labianca R, Haller DG, et al. A pooled analysis of adjuvant chemotherapy for resected colon cancer in elderly patients. N Engl J Med. 2001;345:1091-7.

15. Bergquist JR, Thiels CA, Spindler BA, Shubert CR, Hayman AV, Kelley SR, et al. Benefit of Postresection adjuvant chemotherapy for stage III Colon Cancer in octogenarians: analysis of the National Cancer Database. Dis Colon Rectum. 2016;59:1142-9.

16. Sanoff HK, Carpenter WR, Sturmer T, Goldberg RM, Martin CF, Fine JP, et al. Effect of adjuvant chemotherapy on survival of patients with stage III colon cancer diagnosed after age 75 years. J Clin Oncol. 2012;30:2624-34.

17. Watanabe T, Muro K, Ajioka Y, Hashiguchi Y, Ito Y, Saito Y, et al. Japanese Society for Cancer of the Colon and Rectum (JSCCR) guidelines 2016 for the treatment of colorectal cancer. Int J Clin Oncol. 2018;23:1-34.

18. Kusumoto T, Sunami E, Ota M, Yoshida K, Sakamoto Y, Tomita N, et al. Planned safety analysis of the ACTS-CC 02 trial: a randomized phase III trial of S-1 with Oxaliplatin versus Tegafur and uracil with Leucovorin as adjuvant chemotherapy for high-risk stage III Colon Cancer. Clin Colorectal Cancer. 2018;17:e153-61.

19. Yamano T, Yamauchi S, Kimura K, Babaya A, Hamanaka M, Kobayashi M, et al. Influence of age and comorbidity on prognosis and application of adjuvant chemotherapy in elderly Japanese patients with colorectal cancer: a retrospective multicentre study. Eur J Cancer. 2017;81:90-101.

20. Hamaguchi T, Shirao K, Moriya Y, Yoshida S, Kodaira S, Ohashi Y. Final results of randomized trials by the National Surgical Adjuvant Study of colorectal Cancer (NSAS-CC). Cancer Chemother Pharmacol. 2011;67:587-96.
21. Sadahiro S, Tsuchiya T, Sasaki K, Kondo K, Katsumata K, Nishimura G, et al. Randomized phase III trial of treatment duration for oral uracil and tegafur plus leucovorin as adjuvant chemotherapy for patients with stage IIB/III colon cancer: final results of JFMC33-0502. Ann Oncol. 2015; 26:2274-80.

22. Young JL Jr, Roffers SD, Ries LAG, Fritz AG, Hurlbut AA. SEER summary staging manual 2000: codes and coding instructions. In: Surveillance, Epidemiology, and End Results Program: National Cancer Institute; 2012. https://seer.cancer.gov/tools/ssm/ssm2000. Accessed 23 Dec 2019.

23. Hamada H, Sekimoto M, Imanaka Y. Effects of the per diem prospective payment system with DRG-like grouping system (DPC/PDPS) on resource usage and healthcare quality in Japan. Health Policy. 2012;107:194-201.

24. Morishima T, Matsumoto Y, Koeda N, Shimada H, Maruhama T, Matsuki D, et al. Impact of comorbidities on survival in gastric, colorectal, and lung Cancer patients. J Epidemiol. 2019;29:110-5.

25. Edge SB, Compton CC. The American joint committee on Cancer: the 7th edition of the AJCC cancer staging manual and the future of TNM. Ann Surg Oncol. 2010;17:1471-4.

26. Quan H, Sundararajan V, Halfon P, Fong A, Burnand B, Luthi JC, et al. Coding algorithms for defining comorbidities in ICD-9-CM and ICD-10 administrative data. Med Care. 2005;43:1130-9.

27. Quan H, Li B, Couris CM, Fushimi K, Graham P, Hider P, et al. Updating and validating the Charlson comorbidity index and score for risk adjustment in hospital discharge abstracts using data from 6 countries. Am J Epidemiol. 2011;173:676-82.

28. Shah S, Vanclay F, Cooper B. Improving the sensitivity of the Barthel index for stroke rehabilitation. J Clin Epidemiol. 1989;42:703-9.

29. Yasunaga H, Horiguchi H, Kuwabara K, Matsuda S, Fushimi K, Hashimoto H, et al. Outcomes after laparoscopic or open distal gastrectomy for early-stage gastric cancer: a propensity-matched analysis. Ann Surg. 2013;257:640-6.

30. Lunceford JK, Davidian M. Stratification and weighting via the propensity score in estimation of causal treatment effects: a comparative study. Stat Med. 2004;23:2937-60.

31. Austin PC, Stuart EA. Moving towards best practice when using inverse probability of treatment weighting (IPTW) using the propensity score to estimate causal treatment effects in observational studies. Stat Med. 2015; 34:3661-79.

32. van Buuren S. Multiple imputation of discrete and continuous data by fully conditional specification. Stat Methods Med Res. 2007;16:219-42.

33. Gao Z, Wang C, Cui Y, Shen Z, Jiang K, Shen D, et al. Efficacy and safety of complete Mesocolic excision in patients with Colon Cancer: three-year results from a prospective, nonrandomized, double-blind. Controlled Trial Ann Surg. 2018. https://doi.org/10.1097/SLA.0000000000003012.

34. Hohenberger W, Weber K, Matzel K, Papadopoulos T, Merkel S. Standardized surgery for colonic cancer: complete mesocolic excision and central ligation technical notes and outcome. Color Dis. 2009;11:354-64.

35. Yang Y, Wang G, He J, Zhang J, Xi J, Wang F. High tie versus low tie of the inferior mesenteric artery in colorectal cancer: a meta-analysis. Int J Surg. 2018;52:20-4.

36. Hashiguchi $Y$, Hase $K$, Ueno H, Shinto E, Naito Y, Kajiwara Y, et al. Impact of race/ethnicity on prognosis in patients who underwent surgery for colon cancer: analysis for white, African, and east Asian Americans. Ann Surg Oncol. 2012;19:1517-28.

37. Phatak UR, Kao LS, Millas SG, Wiatrek RL, Ko TC, Wray CJ. Interaction between age and race alters predicted survival in colorectal cancer. Ann Surg Oncol. 2013;20:3363-9.

38. Iwamoto M, Nakamura F, Higashi T. Monitoring and evaluating the quality of cancer care in Japan using administrative claims data. Cancer Sci. 2016;107:68-75.

39. Mathoulin-Pelissier $S$, Becouarn $Y$, Belleannee $G$, Pinon E, Jaffre A, Coureau $\mathrm{G}$, et al. Quality indicators for colorectal cancer surgery and care according to patient-, tumor-, and hospital-related factors. BMC Cancer. 2012;12:297.

40. Khrizman P, Niland JC, ter Veer A, Milne D, Bullard Dunn K, Carson WE 3rd, et al. Postoperative adjuvant chemotherapy use in patients with stage I/III rectal cancer treated with neoadjuvant therapy: a national comprehensive cancer network analysis. J Clin Oncol. 2013;31:30-8.

41. Benson AB 3rd, Venook AP, Cederquist L, Chan E, Chen YJ, Cooper HS, et al. Colon Cancer, version 1.2017, NCCN clinical practice guidelines in oncology. J Natl Compr Cancer Netw. 2017;15:370-98.

42. Labianca R, Nordlinger B, Beretta GD, Mosconi S, Mandala M, Cervantes A, et al. Early colon cancer: ESMO clinical practice guidelines for diagnosis, treatment and follow-up. Ann Oncol. 2013;24(Suppl 6):64-72. 
43. Lembersky BC, Wieand HS, Petrelli NJ, O'Connell MJ, Colangelo LH, Smith $\mathrm{RE}$, et al. Oral uracil and tegafur plus leucovorin compared with intravenous fluorouracil and leucovorin in stage II and III carcinoma of the colon: results from National Surgical Adjuvant Breast and bowel project protocol C-06. J Clin Oncol. 2006;24:2059-64.

\section{Publisher's Note}

Springer Nature remains neutral with regard to jurisdictional claims in published maps and institutional affiliations.

Ready to submit your research? Choose BMC and benefit from:

- fast, convenient online submission

- thorough peer review by experienced researchers in your field

- rapid publication on acceptance

- support for research data, including large and complex data types

- gold Open Access which fosters wider collaboration and increased citations

- maximum visibility for your research: over $100 \mathrm{M}$ website views per year

At $B M C$, research is always in progress.

Learn more biomedcentral.com/submissions 